\title{
Rursus
}

Russus

Poiétique, réception et réécriture des textes antiques

$6 \mid 2011$

Relire, récrire, prolonger. Adaptations gréco-latines

\section{Une méthode Assimil pour apprendre le latin à l'époque humaniste : les Colloquia dérivés du Vocabulare de Noël de Berlaimont}

Béatrice Charlet-Mesdjian et Jean-Louis Charlet

\section{(2) OpenEdition \\ Journals}

Édition électronique

URL : http://journals.openedition.org/rursus/495

DOl : $10.4000 /$ rursus. 495

ISSN : 1951-669X

Éditeur

Université Nice-Sophia Antipolis

Référence électronique

Béatrice Charlet-Mesdjian et Jean-Louis Charlet, « Une méthode Assimil pour apprendre le latin à l'époque humaniste : les Colloquia dérivés du Vocabulare de Noël de Berlaimont », Rursus [En ligne], 6 | 2011, mis en ligne le 01 février 2011, consulté le 19 avril 2019. URL : http:// journals.openedition.org/rursus/495 ; DOI : 10.4000/rursus.495

Ce document a été généré automatiquement le 19 avril 2019

Rursus 


\title{
Une méthode Assimil pour apprendre le latin à l'époque humaniste : les Colloquia dérivés du Vocabulare de Noël de Berlaimont
}

\author{
Béatrice Charlet-Mesdjian et Jean-Louis Charlet
}

En 1530, un flamand de Flandre maintenant française, Noël de Berlaimont (ou Berlaymont), professeur à Anvers, publie un Vocabulare français / flamand connu aujourd'hui par une seconde édition posthume (il est mort en 1531) : Anvers (Antwerpen), W. Vosterman, 1536. L'ouvrage fut rapidement utilisé dans les écoles ${ }^{1}$. En 1551 est imprimée à Louvain, chez Bartholomé De Grave, une version élargie à deux nouvelles langues, l'espagnol (castillan) et le latin, mais encore limitée à trois dialogues. La version latine est due à Cornelius Valerius, professeur au Collège Trilingue de Louvain et maître de Juste Lipse ; le castillan, à deux hispanophones non précisés ${ }^{2}$. A partir de cette date, le livre va connaître un immense succès de librairie, des développements (seulement trois dialogues à l'origine ; cinq à partir de 1579 et sept à partir de 1583) ${ }^{3}$ et de nombreux élargissements aux diverses langues européennes pour aboutir à des versions octolingues imprimées dans toute l'Europe (Pays-Bas y compris la Belgique, France, Suisse, Germanie, Italie, Pologne et Angleterre) jusqu'au milieu du XVIIIe siècle : plus de 150 éditions ${ }^{4}$. Sont successivement introduits :

- l'italien en 1558

- l'anglais et l'allemand en 1576

- le portugais en $1598^{5}$

- et exceptionnellement, par substitution à l'une des langues vernaculaires, le tchèque (Leipzig, 1602) et le polonais (Varsovie, 1646).

Retiré de quelques éditions entre 1558 et 1585, le latin reparaît en 1586 (Anvers, Joachim Trognaesius) ${ }^{6}$ explicitement comme la langue la plus importante. Les deux épîtres latines qui accompagnent cette édition, et qui seront jugées suffisamment importantes pour figurer dans les éditions successives (en particulier Venise, 1656, réimprimée en 1996 et 
qui sera notre édition de référence), sont significatives. Dans l'adresse Liber ad emptores, signée L. B. I. B. F., initiales qui, d'après Verdeyen, signifieraient Ludimagister Bonus Iacobi Boni Filius et désigneraient Assuerus Boon, dont le nom est déjà présent dans une ode accompagnant l'édition de $1579^{7}$, poème hexamétrique de 35 vers, le livre propose à ceux qui ont l'intention de voyager sur «des routes écartées loin de leur patrie» (v. 1-2) d'apprendre chez eux (domi, v. 11) les langues de l'Europe et d' " absorber avec la langue les gestes et les façons de vivre qui conviennent » (v. 15-16) ${ }^{9}$. Guidés par lui (le livre qui prendra la nationalité de chacun de ces pays), ils feront un beau voyage en Flandre, Germanie, France, Angleterre, Espagne, Italie et Portugal (v. 17-26: belle invitation au voyage par la lecture d'un livre!). Les lecteurs pourront participer aux réunions des savants, dans lesquelles il faut changer de langue (langue de culture contre langue usuelle) et leur bouche enfantera chaque jour les beautés du latin (v. 27-29: utilisation culturelle du latin bien attestée à l'époque; mais, comme nous le verrons plus loin, ce n'est qu'un des usages du latin) ${ }^{10}$. Plus explicite encore à propos du latin est la lettre en prose Benevolo lectori datée des ides de novembre (= le 13) 1585 et due à Francisco de Villalobos ${ }^{11}$ : le livre est destiné à ceux qui veulent apprendre les langues (utilisation pédagogique comme méthode d'apprentissage éventuellement gratuit: le plaisir d'apprendre des langues) ou bien voyager (utilité, voire nécessité des langues ; ici, manuel utilitaire). Mais, dans la version précédente en six langues, il manquait l'anglais et le latin omnium potissima Latina lingua. On voit qu'à la fin du XVIe siècle la montée en puissance de l'Angleterre dans le concert européen avec le règne d'Elisabeth rend l'anglais plus important; pendant longtemps, comme l'attestent les éditions plurilingues du Calepino, compte tenu du poids économique et culturel des Pays-Bas le flamand l'emportait sur l'anglais. On a donc remédié à ce manque. A partir de cette date (1586), alors qu'auparavant il peut être placé au centre, entre les langues anglo-saxonnes et les langues romanes, le latin se trouvera pratiquement toujours en tête des langues (première colonne de gauche).

3 Que signifie cette place? Contrairement à $\mathrm{M}$. Furno ${ }^{12}$, nous pensons d'abord que les Colloquia, comme leur titre l'indique, sont d'abord des manuels pour apprendre la langue parlée quotidienne et seulement à titre tout à fait secondaire des lexiques. Outre la part limitée du lexique par rapport aux dialogues ${ }^{13}$, ce lexique est dans l'ordre alphabétique du flamand, ce qui n'était probablement pas très déroutant pour les germanophones, mais certainement pour les autres (français, anglais, italiens, espagnols, portugais...): comment retrouver un mot à partir de sa langue maternelle ou du latin? On ajoutera que cet ordre flamand est conservé même dans les éditions qui ne donnent pas le flamand : en ce cas, seuls les germanophones pouvaient à peu près s'y retrouver ${ }^{14}$ !

Ensuite, si les Colloquia n'ont pas pour but d'apprendre à lire des œuvres littéraires latines, françaises, espagnoles... ni à écrire en ces langues des morceaux de rhétorique ou des dissertations historiques ou morales, ils n'intègrent pas le latin pour donner un air de science à des lecteurs ayant seulement une teinture de cette langue, mais ils visent soit à faire apprendre, à côté d'autres langues, le latin parlé de la vie quotidienne (pour des voyageurs, marchands, soldats...), soit, pour ceux qui connaissent déjà le latin, à l'utiliser comme langue de passage, comme pont entre la langue maternelle et les autres langues vernaculaires : par exemple pour aider un anglo-saxon à passer aux langues romanes. Et cette situation est réelle non seulement dans la seconde moitié du XVIe siècle, mais encore dans le premier tiers du XVIIe. Nous en donnerons deux preuves : les dialogues ( colloquia) et lexiques (même association que dans nos Colloquia!) latin - malgache et latin 
- malais annexés aux Petits voyages de M. de Bry, Supplementum nonae partis Indiae Orientalis, hoc est continuatio prioris itineris sive navigationis ab Hollandis et Selandis in Indiam Orientalem sub admirale Petro Guilhelmo Verhuffio... ab anno 1607 vsque ad annum 1612 peractae (Francfort, veuve de Matthias Becker, 1613) ${ }^{15}$ et d'autre part, en 1633, le petit lexique latin - nahuatl (aztèque) annexé au Novus Orbis seu descriptionis Indiae occidentalis libri XVIII de Johannes De Laet ${ }^{16}$. Le premier ouvrage, publié à Francfort pour un public d'abord germanique et néerlandais, compte tenu du sujet et du lieu d'édition, ne propose pas des dialogues et un lexique allemand ou flamand / malais et malgache, mais seulement latin / malais et malgache, ce qui signifie qu'un allemand ou un hollandais (ou tout autre lecteur) ne pouvait utiliser ce manuel de conversation quotidienne qu'à condition de passer par le latin pour apprendre à parler malais ou malgache! Il lui fallait donc une réelle maîtrise du latin, et non une vague teinture de cette langue. Mais, grâce au latin, l'éditeur de Francfort pouvait toucher tous les lecteurs cultivés d'Europe, voire d'Amérique, qui avaient appris le latin et, s'il n'a pas cru bon d'ajouter certaines langues vernaculaires européennes, c'est que ce public lui paraissait en 1613 suffisamment nombreux pour rentabiliser son édition. Le même raisonnement peut s'appliquer au second ouvrage, de 1633. Dans ce livre sur le Nouveau Monde publié à Leyde, l'auteur aurait pu introduire le flamand / néerlandais, l'espagnol ou encore le portugais: le fait qu'il n'y ait que le latin comme langue européenne prouve qu'à cette date le latin est encore considéré comme la langue de culture commune de l'Europe. L'auteur l'affirme même explicitement en déclarant que la langue aztèque (mexicaine) tam communis esse coepit quam in Europa Latina aut in Asia Slavonica. Les élites européennes cultivées, au moins en grande partie, sont donc encore en situation de diglossie. C'est du moins ce que des éditeurs allemands ou néerlandais, financièrement intéressés, dans deux villes majeures de l'édition européenne, considèrent comme la situation linguistique de l'Europe. Mais nous sommes probablement à la limite extrême de cette situation : au milieu du XVIIe siècle, les langues vernaculaires vont s'imposer, en particulier le français, et l'on verra apparaitre en 1651 des dialogues et un lexique (toujours la même association que dans nos Colloquia!) français / malgache ${ }^{17}$.

Ce Vocabulare, appelé par la suite Colloquia et Dictionariolum (otto...) linguarum, est à l'origine un manuel de conversation de la vie quotidienne, une sorte de méthode Assimil destinée, comme nous l'avons vu, aux marchands et voyageurs ainsi qu'à ceux qui veulent apprendre les langues non seulement pour les lire, mais aussi pour les écrire (écrits commerciaux ou juridiques et non littéraires) et pour les parler (ce dernier point est fondamental: nous y reviendrons). L'avis au lecteur (Studioso Lectori) est sur ce point explicite et la Tabula libri détaille le contenu de l'ouvrage :

1) une première partie de huit chapitres

7 -sept dialogues de conversation usuelle, quotidienne = Colloquia

- banquet de dix convives (p. 10)

- transactions commerciales, acheter et vendre (p. 48)

- réclamer une dette (p. 61)

- demander son chemin (p. 67)

- discussions à l'auberge (p. 73)

- conversation au lever du lit (p. 86)

- discussions de marchands (p. 93) avec la liste des chiffres de un à un million, les jours de la semaine et l'expression du temps (pp. 112-113) 
littéraires, mais commerciales ou juridiques), des contrats, des quittances (rédiger des écrits commerciaux et juridiques), p. 114

2) une seconde partie avec un prologue propre (Praefatio,pp. 136-138) qui comporte un petit dictionnaire (glossaire des mots les plus usuels rangés par ordre alphabétique du flamand, même si le flamand n'arrive généralement qu'en troisième position dans l'ordre des langues : latin, français, flamand, allemand, espagnol, italien, anglais et portugais : pp.139-183) ${ }^{18}$. S'y ajoute un memento grammatical (manuel de conjugaison du verbe « avoir » puis du verbe « être», pp. 184-189), et encore un petit traité de prononciation (indispensable pour apprendre à parler une langue), mais limité au français, à l'italien, à l'espagnol (en français pour ces trois langues) et au flamand confondu pour la prononciation, comme souvent à l'époque, avec l'allemand, en italien (p. 197). Préciser la prononciation du latin ne semblait pas nécessaire à cette date, ce qui peut surprendre : la prononciation érasmienne ne s'était pas encore imposée et chacun prononçait le latin comme sa langue maternelle. Peut-être n'a-t-on pas voulu perturber cet usage. Mais il y a des allusions à la prononciation du latin (langue commune de l'Europe) pour expliquer la prononciation du français, de l'italien, de l'espagnol... (nous verrons plus loin une autre explication). Avec quelques indications grammaticales, très limitées, relatives aux mêmes langues (genre, articles, pronoms), pp. 190-199 et, pour finir, en français, une liste des formations de diminutifs en français, italien, espagnol et flamand (p. 200). En fait, cette dernière section (pp. 190-199) reprend les deux tiers de la seconde partie d'un petit traité du maître anversois Gabriel Meurier (c. 1513-1597) ${ }^{19}$ publié en 1558 à Anvers par Jan van Waesberghe avec le titre bilingue (français / flamand) de :

«Breve Instruction contenante la maniere de bien prononcer et lire le François, Italien, Espagnol et Flamen »

«Korte Instructie inhoudende de maniere om wel te prononceren ende te lesen Franchois, Italiaensch, Spaensch ende Nederduytsch».

12 Ainsi s'explique la limitation à quatre langues de cette troisième partie ajoutée après coup, à partir de l'édition quadrilingue (sans latin: autre raison possible de l'absence relevée plus haut) imprimée par G. de Salenson à Gand en $1568^{20}$. On notera que le même Meurier s'était servi des Colloquia de 1556 pour constituer sa liste des Adverbia, selon un échange de bons procédés fréquent à l'époque (et encore de nos jours pour les manuels scolaires ou de vulgarisation!).

13 Initialement destiné à la conversation de tous les jours (par exemple permettre à un marchand allemand et à un marchand espagnol de se comprendre en parlant tous deux latin, ou en parlant chacun la langue de l'autre ou en comprenant chacun la langue de l'autre), ce manuel permettait aussi d'utiliser le latin pour prendre contact avec des personnes cultivées ou importantes. Pour un italien, un espagnol ou un français, il était peut-être plus commode de parler latin avec un marchand flamand ou allemand plutôt que flamand ou allemand.

14 Du point de vue pédagogique, la méthode est fondée sur le plaisir et sur la répétition. Il n'est pas nécessaire d'insister sur le second principe : toute pédagogie est fondée sur la répétition. C'est ainsi que les expressions les plus usuelles de la vie quotidienne sont systématiquement répétées soit par un jeu de questions / réponses, soit par la structure de dialogue : Vt vales? Bene valeo...Vt valent pater et mater tua ? Bene valent. 

s'apprendre en s'amusant, avec plaisir. C'est pourquoi la mise en scène de chaque dialogue est soignée : chacun constitue une petite scène de comédie destinée à amuser le lecteur tout en lui faisant apprendre les langues. En guise d'exemple, et en nous limitant aux textes latins et français (donnés dans la version d'époque), nous prendrons le premier dialogue Convivium decem personarum («Un convive de dix personnages ») ${ }^{21}$. Le dialogue met en scène dix acteurs, avec des entrées successives. Un premier dialogue entre deux condisciples, Hermès et Jean, nous apprend, vers midi, qu'Hermès a fait la grasse matinée au lieu d'aller, comme Jean, à l'école. Puis Jean regagne, en retard, sa maison : sa mère Marie lui reproche d'arriver à six heures, alors qu'elle l'attendait à quatre, et lui demande de mettre la table ${ }^{22}$. C'est l'occasion d'introduire dans le dialogue tout le vocabulaire correspondant. Jean fait traîner les choses en feignant d'ignorer où sont les objets qu'on lui demande de placer. Sa mère préfère alors lui demander d'aller chercher le pain, en prenant soin de distinguer les deux sortes de pain : le «pain blanc» (panis candidus) et le « pain gris » (panis cibarius), et en indiquant leur prix respectif. De retour, Jean doit aller chercher du bois pour allumer le feu. Arrive son père, Pierre, avec un cousin, David. Après un échange de formules de politesse (du genre : Exoptatus ades, mi pater, cum tuis sodalibus), David interroge son oncle sur les études de Jean qui apprend... le français ${ }^{23}$ ! Marie, tante de David, lui demande pourquoi il n'est pas venu avec sa sœur : elle est malade. On frappe à la porte. François, frère de Jean, va voir qui c'est. C'est Roger, un ami de la famille (dialogue de reconnaissance à la porte). Le père, Pierre, appelle toute la famille à table. S'en suit une scène de comédie pour faire accepter à chacun sa place. Puis on récite une sorte de benedicite, religieux en langues vernaculaires, plus laïc en latin (1996, p. 23a) :

Felix faustumque sit vobis, pater ac mea mater, totique sodalitio.

Dieu vous benie, mon pere, ma mere, et toute la compagnie.

Le repas peut alors commencer : énumération des nombreux plats et recommandations (que l'on entend encore aujourd'hui !) des parents aux enfants et même de la mère, Marie, à son mari Pierre (1996, p. 25a) :

Petre, ne mox bibas a sorbitione, est animi insalubre : prius aliquid ede quam bibas.

Pierre, ne beuvez point après vostre potage, car il est malsain : mangez premier un peu devant que vous beuviez.

Enervé, ledit mari refuse de servir ses enfants comme sa femme le lui demande : ils sont assez grands pour se servir tout seuls! Puis la femme réprimande son fils d'avoir renversé du vin en servant son père. Survient un nouveau convive, Anna. Les plats continuent de défiler et le vin de couler jusqu'au moment où il fait défaut: Pierre envoie alors son fils Jean en chercher, et l'on passe en attendant à la "cervoise» (cervisia). On frappe à nouveau à la porte : c'est Henri, le serviteur de l'oncle de Pierre, qui lui apporte une invitation à "disner » (= déjeuner : in prandio) pour le lendemain midi. Pierre invite à boire Henri, qui tente de refuser. Survient Jean avec le vin qu'il vient d'acheter. On discute pour savoir s'il est meilleur que le précédent. Mais on s'étonne du silence d'Anna : celle-ci dit ne pas parler parce que sa connaissance du français serait insuffisante! Au passage, le dialogue atteste une pratique linguistique qui durera jusqu'au XXe siècle et qui produira en Flandre la réaction politique violente des Flamingants que l'on connaît : une partie de la bourgeoisie flamande se pique de parler français chez elle ${ }^{24}$. Suivent divers propos de table : discussion à propos d'un couteau, sur la nourriture, à propos des toasts que l'on a portés (le verre était-il bien plein? Anna, qui semble un peu la souffre-douleur, 
a-t-elle réellement bu à la santé de Marie ? ...). Et l'on apporte encore de nouveaux plats ! Une dernière personne vient frapper à la porte: Lucas, le serviteur d'André, ami de Pierre, qui apporte un cadeau de la part de son maître. On lui verse à boire et l'on allume les chandelles. La conversation tourne sur la situation militaire: les Français ont-ils réellement, comme on le rapporte, été battus par les Espagnols ? La paix est-elle proche? Comme au café du commerce, mais non sans une certaine sagesse populaire, on donne des leçons de diplomatie (1996, p. 45a-46a) :

Quod si nos vellemus cum eo [le roi de France] pacem facere, bellum non foret diuturnum.

Si nous voulions faire paix avec luy, la guerre ne dureroit pas longuement.

Pour finir, on dit les grâces... en latin puisque, dans toutes les versions vernaculaires, on lit la formule latine: De tali convivio, et cetera. On boit à nouveau, ce qui donne l'occasion de réitérer les grâces. Roger veut payer à Pierre le vin qu'il a bu : le vin est cher $^{25}$ ! Mais Pierre refuse : un invité ne paie pas! Il est alors 10 heures du soir et les invités prennent congé de façon laïque en latin... et de façon religieuse dans les langues vernaculaires (1996, p. 48a) :

$H($ (enricus) : Precor vobis felicem noctem. - P(etrus) : Nosque vobis. Valete.

H. : Dieu vous doint bonne nuit. - P. Et à vous aussi, à Dieu vous commande.

Comme on le voit, la mise en scène, qui suppose parfois des coupures temporelles (par exemple ici entre midi et six heures du soir), n'est pas seulement utilitaire : l'intrigue permet certes d'introduire tout le vocabulaire et toutes les expressions qui correspondent à une situation donnée (parfois avec des expressions de la comédie latine, parfois avec des mots propres au néo-latin pour désigner des réalités contemporaines). Mais c'est un dialogue vivant, parfois cocasse, qui présente sous forme comique (comique de situation) des scènes de la vie quotidienne. Le lecteur s'amuse beaucoup en apprenant.

Ces Colloquia sont donc des témoins privilégiés de ce que pouvait être le latin parlé (non le latin lettré, le latin religieux ou le latin scientifique) aux XVI-XVIIe et même XVIIIe siècle, parallèlement aux langues vernaculaires, dans certaines couches instruites de la population. Grâce aux sept et même neuf langues vernaculaires européennes qu'il contient selon les versions (témoignage précieux pour les formes parlées de ces langues à la Renaissance!), ce type de manuel permet des comparaisons éclairantes entre les langues européennes et le latin à l'époque humaniste et postérieure. Il a aussi été utilisé à partir de la seconde moitié du XVIe siècle pour l'apprentissage du latin à l'école: n'oublions pas que, jusqu'au XVIIIe siècle (et même encore parfois jusqu'au début du XXe siècle), le latin y est enseigné comme une langue vivante que l'on parle et non comme une langue morte dont on se contente de lire les témoignages écrits. L'étude de ce manuel pourrait aussi apporter un éclairage intéressant sur la pédagogie du latin et des langues européennes de 1550 à 1750. C'est d'ailleurs dans une perspective de didactique de l'espagnol (castillan) que des collègues de l'université de Pise se sont intéressés à ce texte et en ont fourni une réédition moderne en 1996. 


\section{BIBLIOGRAPHIE}

ALCINA RovinA, J. F., «El latín humanístico y la cultura vernácula de los siglos de oro », in AVILA Alonso et al., Filología latina hoy: Actualización y perspectivas, t. II, Madrid, Sociedad de Estudios Latinos, 1999, pp. 729-746

BINGEN N., Le Maître italien (1510-1660), Bruxelles, Emile Van Balberghe, 1987

BOURLAND C., "The Spanish School-master and the polyglot derivatives of Noel de Berlaimont's

Vocabulare ", Revue hispanique,81,1, 1933, pp. 283-318

CARAVOLAS Jean Antoine, La didactique des langues. Précis d'histoire, I : 1450-1700, Montréal /

Tübingen, Presses de l'université de Montréal / Gunther Narr, 1994

FURNO Martine, « Du commerce et des langues : latin et vernaculaires dans les lexiques et dictionnaires plurilingues du XVIe siècle », Histoire et civilisation du livre. Revue internationale (Droz), 4, 2008, pp. 93-116

HOOCK J. - JEANNIN P., Ars Mercatoria. Eine analytische Bibliographie, Paderborn-München-WienZürich, Schöning, B. 1 (1470-1600), 1991 ; B. 2 (1600-1700), 1993 ; B. 3 Analysen 1470 - 1600), 2001

LE GOAZIOU A., La longue vie de deux 'Colloques François et Breton' (1626-1915), Quimper, librairie Le Goaziou, 1950

PETRUCCI Armando, « Alle origini del libro moderno. Libri da banco, libri da bisaccia, libretti da mano ", IMU,12, 1969, pp. 295-313 [ = pp. 139-156 de Libri, scrittura e pubblico nel Rinascimento, Bari, Laterza, 1979]

PFISTER M., «Latein und Volkssprache in der Lexicographie des 15. und 16. Jhs. Tradition und Innovation ", in E. Ecker - C. Zintzen (éd.), Caesulum tamquam aureum. Internationales Symposion zur italienischen Renaissance des 14.-16. Jahrhunderts am 17. / 18. September 1996 in Mainz, Hildesheim, Olms, 1997, pp. 303-321

REBOUILLET A., « Guillaume Quiquer et le français langue étrangère en Basse-Bretagne », Documents pour l'histoire du français langue étrangère ou seconde,22, 1998, pp. 64-71

RIZZA Riccardo (a cura di), Colloquia et Dictionariolum Octo Linguarum, Latinae, Gallicae, Belgicae, Teutonicae, Hispanicae, Italicae, Anglicae, Portugallicae, [réimpression de Venise, 1646] ViareggioLucca, Mauro Baroni, 1996 ROSSEBASTIANO-BART Alba, "I 'Colloquia' di Noel de Berlaimont nella versione contenente il portoghese », Annali dell'Istituto Universitario Orientale, Sezione Romanza, 17, 1975, pp. 31-85 EAD., Antichi vocabulari plurilingui d'uso popolare : la tradizione del 'Sollenissimo Vocabuolista', Alessandria, Edizioni dell'Orso, 1984

TIMELLI Maria Colombo, « Dictionnaires pour voyageurs, dictionnaires pour marchands - ou la polyglossie au quotidien aux XVIe et XVIIe siècles », Linguisticae Investigationes,16,2, 1992, pp. $395-420$

EAD., « Dialogues et phraséologie dans quelques dictionnaires plurilingues du XVIe siècle (Berlaimont et Solenissimo Vochabulista) », Documents pour l'histoire du français langue étrangère ou seconde,22, 1998, pp. 27-63 
EAD. - REBOULLET A., « Parcours professionnels au XVIe siècle. Berlaimont, Hollyband, Meurier », Le français dans le monde, numéro spécial (Histoire de la diffusion et de l'enseignement du français dans le monde), 1998, pp. 21-23

EAD. , « Aspetti didattici nei dizionari plurilingui del XVI-XVII secolo : il 'Berlaimont' ", Lessicologia e lessicografia nella storia degli insegnamenti linguistici, Bologna 15/15-11-2003 = Quaderni del CIRSIL 2, 2003, www. lingue.unibo.it/cirsil, 11 pages

VERDEYEN W. R. R., Colloquia et Dictionariolum septem linguarum [réimpression d'Anvers 1616], Antwerp, Uitgave van de Vereeniging der Antwerpsche Bibliophilen, 3 vol. , 1925-1935

WAENTIG Peter W., «Die Venezianische Ausgabe der Colloquia et dictionariolum octo linguarum : Editionsgeschichte - Werkstruktur - Sprachkultur », in W. Hüllen et F. Klippel (éd.) Heilige und profane Sprachen... Die Anfänge des Fremdsprachenunterrichts im westlichen Europa, Wiesbaden, Harrassowitz, 2002, « Wolfenbütteler Forschungen » 98, pp. 175-197

ID., Colloquia et dictionariolum octo linguarum. Tedesco protomoderno. Edizione e commento della versione del 1656, Bologna, Clueb, 2003

ID., « Gesprächsbücher bi- e plurilingui nell'Europa occidentale tra il Trecento ed il Seicento : aspetti lessicologico-lessicografici della terminologia tessile », Lessicologia e lessicografia nella storia degli insegnamenti linguistici, Bologna 15/15-11-2003 = Quaderni del CIRSIL 2, 2003, www.

lingue.unibo.it/cirsil, 16 pages

\section{NOTES}

1. Le maître d'école anversois Glaude (Claude ?) Luython, originaire de Flandre française, l'utilisa comme texte scolaire en 1537 (Rizza 1996: p. VI d'après Verdeyen 1925-1935). On peut aussi penser que Berlaimont lui-même, professeur de français, fut le premier à utiliser cette méthode dans son enseignement.

2. Rizza 1996 : p. VII.

3. Nous mentionnerons plus loin d'autres ajouts.

4. D'après le décompte de R. Verdeyen 1925-1935. Il existe d'autres dictionnaires plurilingues, par exemple celui de Francesco Garone (voir Furno 2008 : 95, n. 6 : Quinque linguarum vocabulista, Lyon, Jacques Moderne, 1542 ; latin, italien, français, espagnol, allemand) ou le Dictionarium quatuor linguarum (flamand, français, latin et espagnol) publié à Louvain en 1556 chez Bartholomé de Grave (avis au lecteur en latin de Francisco Villalobos, correcteur hispanisant; Furno 2008 : 95, n. 7 et 8) et d'autres manuels de conversation comme celui d'Hermann Schotten de Hesse, qui, sans craindre de venir après Erasme, a écrit ses Colloquia tout en latin(quotidiani sermonis formulas) pour ses élèves et pour les débutants, le premier dialogue, entre Albertus et Antonius, étant intitulé Difficile esse Latinum discere sermonem (j'ai consulté à la B. M. de Lyon les deux éditions lyonnaises chez Theobald Paganus, 1545 et 1566, respectivement B 511956 et B 510183 ; format $10,5 \mathrm{~cm} \times 15,5 \mathrm{~cm})$.

5. Voir Bart Rossebastiano 1975. C'est l'année de la fondation de la synagogue d'Amsterdam, peut-être liée à l'importante communauté juive portugaise des Pays-Bas.

6. Rizza 1996 ; mais Furno (2008: 100) dit « en 1583 ».

7. Est-ce lui qui a fait les versions latines des chapitres 4 à 7 absents des premières éditions, ou Francisco de Villalobos plusieurs fois mentionné?

8. «Vos quibus est animus patrio procul orbe remotas / Ire vias».

9. «Simul et gestus moresque decoros / Cum lingua imbibere». 
10. «Quin ubi Palladios inter consistere coetus, / Et vacat alternare sonos, tunc divite vena / Parturiet Latios dies mihi lingua lepores" .

11. Le Villalobos mentionné plus haut (Furno 2008 : 95).

12. Furno 2008 : 97-101 et 112-113 (conclusions d'une étude plus adaptée au Vocabulista quinque linguarum de Garone, 1542, et à certains autres manuels de ce type qu'aux Colloquia qui nous intéressent ici).

13. Trois pages de préface et 47 pages de lexique contre 124 pages de dialogues dans l'édition 1646 / 1996.

14. Vérification faite sur l'édition en six langues de Genève, Iacobus Stoer, 1651 (exemplaire de la bibliothèque Sainte-Geneviève, Réserve 593 X 311).

15. Exemplaires consultés: Lyon, bibliothèque municipale, Rés. 158107 ; Paris, bibliothèque Sainte-Geneviève, Réserve FOL G 171 INV 217 RES (P. 4).

16. Lugduni Batavorum [Leyde], Elzevier, 1633, p. 240.

17. Relations veritables et curieuses de l'Isle de Madagascar et du Bresil Auec l'Histoire de la derniere Guerre faite au Bresil, entre les Portugais \& les Hollandois. Trois relations d'Egypte \& une du royaume de Perse, Paris, Augustin Courbé, 1651 (achevé d'imprimer le 10 septembre 1651). Cet ouvrage (consulté dans la bibliothèque de Christian Charlet contient, annexés à la Relation du voyage que François Cauche de Roüen a fait en l'Isle de Madagascar, des "Colloques" entre un Malgache ( Madagascarois ») et un Français « sur les choses les plus nécessaires pour se faire entendre et estre entendu d'eux. Le tout recüeilly par le sieur Morisot auec des notes en marge». Les Colloques vont de la p. 175 à la p. 190 et sont suivis par un petit lexique de trois pages (p. 191-193) contenant «quelques mots significatifs des choses les plus necessaires» (exemples: "Loha la teste, Voule les cheveux »).

18. Quand on compare dans le détail les versions des différentes langues (comparaison faite sur le premier dialogue), on s'aperçoit que le français et le flamand sont très liés dans leur formulation (ce qui correspond aux premières versions bilingues de ces Colloquia), de même que de leur côté le latin et l'allemand (l'allemand aurait été traduit à partir du latin ?). Pour les quatre autres langues (nous n'avons pas pu consulter les éditions donnant le tchèque et le polonais... et nous n'avons pas de compétence dans ces deux langues slaves!), les accords sont plus fluctuants.

19. Rizza 1996 : IX (Furno -2008 : 95- mentionne Robert Estienne 1546).

20. Peut-être déjà en 1556 à Louvain (Furno 2008 : 95).

21. Jean-Louis Charlet a présenté le septième dialogue (Collocutiones ad mercaturam pertinentes, Propos de marchandise) devant le Groupe Numismatique du Comtat et de Provence (Avignon, 7 février 2010). Cette communication sera publiée dans le volume 2010 [2011] des Annales de ce groupe sous le titre: "Les monnaies sur un marché du XVIe siècle d'après les Colloquia ». Ce dialogue contient en effet un passage savoureux dans lequel un acheteur, après avoir longuement discuté le prix d'une pièce de drap sur un marché, tente de la payer : les monnaies qu'il propose au vendeur présentent toutes un défaut (de mauvais poids, de mauvais titre, rognées, démonétisées, falsifiées...). On cherche à épuiser toutes les raisons possibles ou imaginables pour refuser une monnaie. A ce comique d'accumulation (qui correspond à une visée pédagogique) s'ajoute par moments, pour l'amusement du lecteur - élève, la mauvaise foi du vendeur ou de l'acheteur. Quant au premier dialogue analysé ci-dessous, il a fait l'objet de son séminaire au premier semestre de l'année 2009-2010.

22. On dîne tôt, encore de nos jours, dans les pays du nord de l'Europe.

23. On n'oubliera pas que l'auteur des premiers dialogues, Berlaimont, enseignait le français à Anvers.

24. Comme nous l'avons rappelé dans la note précédente, Berlaimont est venu à Anvers enseigner le français à cette bourgeoisie et à ses enfants.

25. Réalité économique dans une région des Pays-Bas (Anvers) qui ne cultive pas la vigne! 


\section{RÉSUMÉS}

Issus du Vocabulare bilingue (français-flamand) de Noël de Berlaimont, les Colloquia sont des méthodes d'apprentissage de la langue parlée : quatre langues (français, flamand, espagnol, latin) et finalement huit avec l'italien, l'anglais, l'allemand et le portugais (exceptionnellement le tchèque et le polonais). Destinés aux voyageurs ou marchands et à ceux qui veulent apprendre des langues sans quitter leur domicile, et même aux écoles, ces Colloquia connaissent un immense succès du milieu du XVIe au milieu du XVIIIe siècle. A partir de la fin du XVIe siècle, le latin y tient la première place : non seulement le latin est encore parlé par une bonne partie de l'élite européenne (situation de diglossie au moins jusqu'au milieu du XVIIe siècle), mais il sert souvent de langue de passage entre la langue maternelle et une autre langue vernaculaire. La pédagogie mise en œuvre se fonde sur la répétition et la mise en scène. Chaque dialogue est une petite comédie vivante et parfois cocasse: le plaisir qu'on y prend facilite l'effort d'apprentissage. Les Colloquia constituent un témoignage privilégié sur les formes parlées du latin et des langues européennes au XVIe siècle et sur leur pédagogie.

Descended from Noel Berlaimont's bilingual Vocabulare (French-Flemish), the Colloquia are didactic methods to learn spoken languages: four tongues (French, Flemish, Spanish and Latin) and finally eight with Italian, English, German and Portuguese (exceptionally Polish and Czech). Written for travellers and merchants but also for anybody who wants to learn languages at home, and even for schools, these Colloquia are a best seller from the middle of the sixteenth century to the middle of the eighteenth. From the end of the sixteenth, Latin has got the first rank : not only is Latin still spoken by a great part of the European Elite (diglossia at least until the middle of the seventeenth century), but also this language is often used as an intermediate language between the native tongue and an other modern language. The pedagogy of this book is based on repetition and scenery : each dialogue is a little vivid and sometimes laughable comedy. The pleasure that a reader took made his effort easier. The Colloquia are precious because they give evidence about the spoken forms of Latin and modern European languages in the sixteenth century, and about the pedagogy of languages.

\section{INDEX}

Mots-clés : apprentissage du latin, Berlaimont, diglossie, latin parlé, manuels plurilingues

Keywords : Berlaimont, diglossia, latin pedagogy, learning latin, multilingual manuals

\section{AUTEURS}

\section{BÉATRICE CHARLET-MESDJIAN}

Université de Nice - Sophia Antipolis

Maître de conférences de latin à l'université de Nice - Sophia Antipolis est l'auteur d'une thèse intitulée Tito Vespasiano Strozzi, poète élégiaque, le saut de Bucéphale ou une esthétique de la mobilité 
(2001, université de Provence). Elle a publié plus de 25 articles en France et à l'étranger. Elle travaille principalement sur la poésie latine humaniste, les genres élégiaque, épigrammatique et bucolique : l'ambiance ferraraise (Tito et Ercole Strozzi, Guarino de Vérone, Janus Pannonius), Pétrarque et E. S. Piccolomini (mise en scène de la comédie Chrysis avec J.-L. Charlet). Elle s'intéresse aussi à la lexicographie latine humaniste et à la linguistique comparée des langues romanes. Elle a participé avec ses collègues niçois à l'édition des Passions d'amour de Parthénios de Nicée.

\section{JEAN-LOUIS CHARLET}

Université de Provence.

Professeur de latin tardif, médiéval et humaniste à l'université de Provence, est spécialiste de poésie latine tardive (articles sur Damase, Ambroise, Ausone, Paulin de Nole, Prudence, Sidoine Apollinaire, Rutilius... ; livres sur Ausone et Prudence ; édition de Claudien dans la Collection des Universités de France), de philologie et lexicographie latines humanistes (nombreux articles sur Valla, Tortelli, Perotti, Maio, Nestore Dionigi, Paolo Pompilio, Grapaldo, Calepino ; édition du Cornu copiae et de la Lettre à Guarnieri de N. Perotti), de poésie et de métrique néo-latines (nombreux articles sur Pétrarque, E. S. Piccolomini, Campano, Landino, Marulle, Politien, Altilio, A. et G. Costanzi, Crinito ; édition de la Chrysis d'E. S. Piccolomini), de l'histoire du livre et aussi de numismatique. 